\title{
The Feasibility of 1940-nm Diode Laser in Tonsillectomy
}

\author{
Karam Kang \\ Hyunjung Kim \\ Jae Hyung Kim \\ Nu-Ri Im \\ Hyun Ji Lee \\ Byoung Jae Kim \\ Kwang Yoon Jung \\ Tae Hoon Kim \\ Seung-Kuk Baek
}

Department of Otorhinolaryngology-Head and Neck Surgery, Korea University College of Medicine, Seoul, Korea
Received December 5, 2016

Revised December 19, 2016

Accepted December 19, 2016

\footnotetext{
Correspondence

Seung-Kuk Baek

Department of Otorhinolaryngology-Head and Neck Surgery, Korea University Anam Hospital, 73 Inchon-ro, Seongbuk-gu, Seoul 02841, Korea Tel.: +82-2-920-6618

Fax: +82-2-920-5233

E-mail: mdskbaek@agmail.com

(C) Korean Society for Laser Medicine and Surgery

(c) This is an open access article distributed under the terms of the Creative Commons Attribution NonCommercial License (http://creativecommons.org/ licenses/by-nc/4.0) which permits unrestricted noncommercial use, distribution, and reproduction in any medium, provided the original work is properly cited.
}

\author{
Background and Objectives \\ Tonsillectomy is a painful procedure that often results in severe pain, \\ postoperative hemorrhage, and swallowing difficulties, jeopardizing the \\ quality of life in patients. We aimed to investigate the effectiveness of the \\ 1940-nm diode laser method compared with the more commonly used \\ electro-cauterization method for tonsillectomy.
}

\section{Materials and Methods}

Twenty-two patients, who underwent tonsillectomy for chronic tonsillitis, were prospectively analyzed. Tonsillectomy was performed using either a 1940-nm laser with $12 \mathrm{~W}$ of power or the conventional electro-cauterization. Patients were randomly assigned to one of the two groups. A 5-point scale (1-5) was used to measure the intraoperative score via the cutting capacity of the device, and a 3-point scale (0-2) to measure intraoperative bleeding (3-point scale, 0-2). Patients' surveys on pain and the surgeon's reports on postoperative changes in the surgical field, such as edema and scarring, were also analyzed (11-point scale, 0 -10) on postoperative one day, one week, and two weeks, respectively.

\section{Results}

The mean operative time was 22.6 minutes. Operation time, cutting capacity, and intraoperative bleeding were not significantly different between the two groups. Pain scores of patients for the laser group were significantly reduced compared with that of electro-cautery group at one week, even if the surgeon's assessments of scar contraction and swelling were not different between the two groups. Moreover, there was less destruction of tonsil tissues in the laser group according to the histological evaluation.

\section{Conclusion}

A 1940-nm diode laser demonstrates excellent intraoperative performance that is similar to the conventional device. Postoperatively, a notable reduction in pain score on postoperative one week was elicited in patients using 1940-nm diode laser.

\section{Key words}

Laser; Tonsillectomy; Pain 


\section{INTRODUCTION}

As the most common surgery performed in otolaryngology, tonsillectomy is one of the agonizing procedures that often leads to severe pain, postoperative hemorrhage, and swallowing difficulties, hence jeopardizing the patient's quality of life. Children often suffer from dehydration, secondary to postoperative pain, that lead to readmission to hospital for intravenous hydration; adults often experience sedation and constipation from narcotic pain medication. 'Many instruments and techniques have developed with sole purpose of reducing aforementioned complications. With the idea of lower temperatures causing less tissue damage, hence diminishing postoperative pain, both the harmonic ultrasonic scalpel and the coblator have arisen as new surgical instruments that may replace electro-cauterization. ${ }^{2}$ Whereas electrocauterization generates heat at temperature $400-600^{\circ} \mathrm{C}$, harmonic ultrasonic scalpel and the coblator generate at 60-100 ${ }^{\circ} .^{3}$

For better hemostasis and wound healing, lasers have been growing of its importance. Initially introduced to urological laparoscopy and benign prostate disease surgery, effectiveness of laser in incision and coagulation is already well proven. ${ }^{4}$ Allowing tissue penetration of 2-3 $\mathrm{mm}$ with water absorption of wavelength at $1,470 \mathrm{~nm}$, the diode laser was primarily used for the treatment of benign prostate hypertrophy and enabled high tissue ablation with excellent hemostasis and minimal tissue distortion. ${ }^{5-7}$ In addition, the beam seals small blood vessels, lymphatic vessels, and nerve endings to create hemostasis, to reduce tumor cell spread, and to reduce postoperative pain, respectively. ${ }^{8}$ Currently $\mathrm{CO}_{2}(10,600 \mathrm{~nm})$, erbium-doped yttrium aluminum garnet laser (Er:YAG; $2940 \mathrm{~nm}$ ), and erbium, chromium-doped yttrium, scandium, gallium, and garnet laser (ER, CR:YSGG; $2780 \mathrm{~nm}$ ) are the most widely used laser types for intraoral surgery.
Herein, we aimed to prove efficacy of diode laser, using wavelengths of $1940 \mathrm{~nm}$, in tonsillectomy compared to widely used electro-cautery device in terms of various standards.

\section{MATERIALS AND METHODS}

\section{Patients}

This study was performed in a single tertiary hospital in Seoul, South Korea. Twenty-two patients were prospectively enrolled from April 2015 to May 2016. All patients were diagnosed with chronic tonsillitis, and were followed up for two weeks after tonsillectomy. The study excluded patients under 20 years of age, as well as patients with insufficient or irregular follow-ups. This study was approved by the Institutional Review Board of Korea University Hospital, Seoul, South Korea (IRB No. MD15001).

\section{Laser system}

A laser system (Xlender-Y; Wontech, Daejeon, South Koreal with wavelength of $1940 \mathrm{~nm}$ was used for the operation (Fig. 1). Attaching the output of a laser diode module to an optical fiber enabled deliver of light to the operation site. The fiber-attached laser diode module offered up to $12 \mathrm{~W}$ of output through a $600-\mu \mathrm{m}$ diameter optical fiber with 0.22 numerical apertures. User interface was used to set parameters for both the output power and exposure time of the laser.

\section{Procedure}

Single surgeon (S.K.B.) performed the operation. Under general anesthesia, patients were placed in supine position. Dingman mouth gag was applied to open the patients' mouth. The laser mode was set at $12 \mathrm{~W}$ of power with continuous mode. The incision was made on the anterior pillar of the tonsil, then the tonsil capsule was identified. Pulling the tonsil medially exposes a plane between
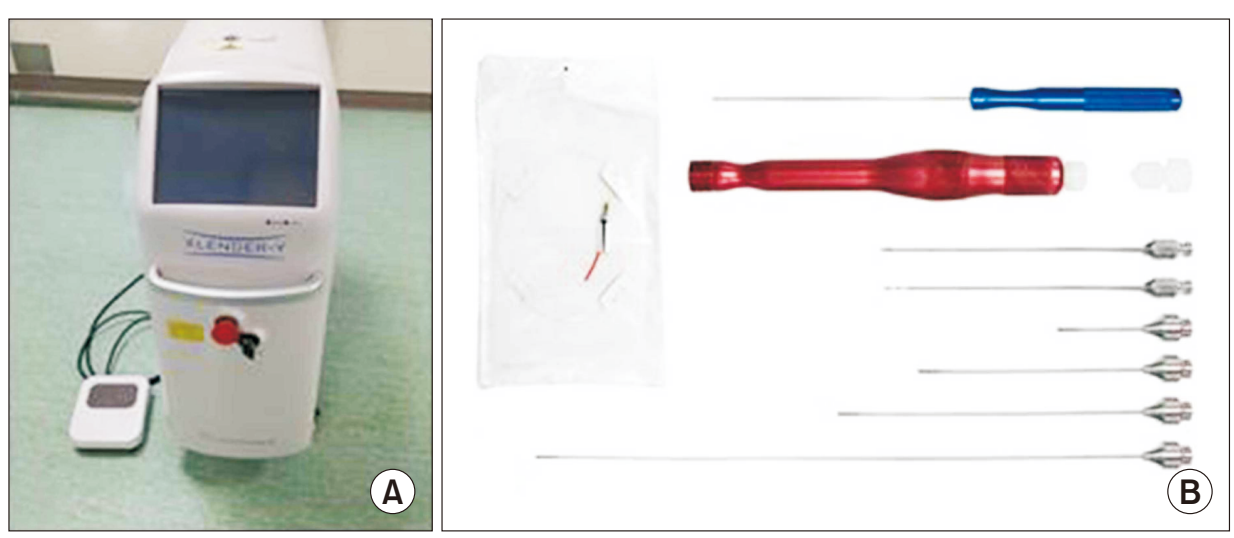

Fig. 1. Laser instruments. (A) Diode laser (Xlender-Y; Wontech) with 1927-nm or 1940-nm wavelengths. (B) $600-\mu \mathrm{m}$ optical fiber with 0.2 numerical aperture and cannulas with various lengths. 
the exposed tonsil capsule and adjacent muscles, and the laser was used to dissect this plane. The laser was set to defaced mode to control mild bleeding during the operation; however, bipolar electro-cautery was used in moderate bleedings where the laser could not effectively stop bleeding. The surgical bed was re-evaluated thoroughly once both tonsils were extracted. Further bleeding control was done if necessary. All patients were discharged on postoperative day one without any complications, and periodic follow-ups were implemented at postoperative one week and two weeks after discharge.

Monopolar electro-cautery device (Force FX'M Electrosurgical Generator C; Covidien, MN, USA) was used as the same procedure to laser tonsillectomy. The electrocauterization mode was $20 \mathrm{~W}$ with blending mode. In addition, bipolar electro-cautery was used in the bleedings where the monopolar electro-cautery device could not effectively coagulate. All patients were discharged on the same day and were taken the same periodic follow-ups as the laser group.

\section{Clinical parameters}

The surgeon's 5-point grading scale was applied to evaluate the cutting capacity of two devices: 1 , insufficient;
2. poor; 3, moderate; 4, good; 5, very good. In terms of intraoperative bleeding, 3-point grading scale was used: 0 , no bleeding; 1 , mild bleeding without requiring the additional application of the device for bleeding control; 2 , bleeding requiring additional procedures. The surgeon's assessment for swelling and scar contraction on postoperative one day, one week, and two weeks were evaluated on a 10-point grading scale. Patients' self-report regarding pain on postoperative one day, one week, and two weeks were evaluated on a 10-point grading scale as well. Moreover, operation time and number of times that bipolar electro-cauterization were used for bleeding control during operation were evaluated.

\section{Histologic evaluation of tissue destruction after tonsillectomy}

The three pieces of tissue were acquired from upper, middle, and lower regions of tonsil that was removed with laser and electro-cauterization. Two consecutive 5- $\mu \mathrm{m}$ sections were cut from each paraffin-embedded block. One section from each specimen was routinely stained with $H \& E$, and another section was stained with Masson's trichrome stain (NovaUltra ${ }^{\mathrm{TM}}$ Masson Trichrome Stain Kit; IHC WORLD, Ellicott City, MD, USA) to estimate the sever-

Table 1. Demographics and intraoperative findings

\begin{tabular}{|c|c|c|c|c|c|c|c|}
\hline No. & Age & Gender & Device & Operation time (min) & $\begin{array}{l}\text { Intraoperative } \\
\text { bleeding }^{*}\end{array}$ & $\begin{array}{c}\text { Number of bipolar } \\
\text { cauterization }\end{array}$ & Cutting capacity ${ }^{\dagger}$ \\
\hline 1 & 26 & $\mathrm{~F}$ & Laser & 15 & 0 & 0 & 4 \\
\hline 2 & 19 & $M$ & Laser & 25 & 1 & 4 & 5 \\
\hline 3 & 45 & $\mathrm{~F}$ & Laser & 20 & 0 & 1 & 5 \\
\hline 4 & 19 & $\mathrm{~F}$ & Laser & 20 & 0 & 1 & 5 \\
\hline 5 & 20 & $\mathrm{M}$ & Laser & 32 & 1 & 0 & 3 \\
\hline 6 & 20 & $\mathrm{~F}$ & Laser & 35 & 2 & 5 & 3 \\
\hline 7 & 54 & $\mathrm{M}$ & Laser & 15 & 0 & 1 & 5 \\
\hline 8 & 19 & $\mathrm{~F}$ & Laser & 20 & 0 & 0 & 5 \\
\hline 9 & 40 & M & Laser & 25 & 0 & 3 & 5 \\
\hline 10 & 50 & $\mathrm{~F}$ & Laser & 25 & 0 & 2 & 4 \\
\hline 11 & 45 & $\mathrm{~F}$ & Laser & 10 & 0 & 2 & 5 \\
\hline 12 & 19 & M & Laser & 20 & 1 & 1 & 4 \\
\hline 13 & 24 & $M$ & Bovie & 25 & 0 & 2 & 5 \\
\hline 14 & 18 & $M$ & Bovie & 20 & 1 & 0 & 5 \\
\hline 15 & 25 & $\mathrm{M}$ & Bovie & 20 & 0 & 1 & 4 \\
\hline 16 & 54 & $\mathrm{~F}$ & Bovie & 25 & 1 & 2 & 5 \\
\hline 17 & 21 & $F$ & Bovie & 20 & 0 & 1 & 4 \\
\hline 18 & 28 & $\mathrm{M}$ & Bovie & 30 & 1 & 1 & 3 \\
\hline 19 & 30 & $\mathrm{~F}$ & Bovie & 30 & 2 & 4 & 4 \\
\hline 20 & 48 & $M$ & Bovie & 20 & 0 & 1 & 4 \\
\hline 21 & 23 & $\mathrm{~F}$ & Bovie & 20 & 1 & 2 & 5 \\
\hline 22 & 37 & $F$ & Bovie & 25 & 2 & 4 & 5 \\
\hline
\end{tabular}

$* 0$ = no bleeding, $1=$ mild bleeding without requiring additional appliance of laser for bleeding control, $2=$ bleeding requiring additional procedure; ${ }^{\dagger} 1$ = insufficient, $2=$ poor, $3=$ moderate, $4=$ good, $5=$ very good. 
ity of tissue destruction resulted from two devices. After mounted using mounting medium, slides were examined with an Olympus BX51 microscope lOlympus Corp., Tokyo, Japan). Pictures were captured and controlled in Olympus DP72 and DP2-BSW (Olympus Corp.).

\section{RESULTS}

Table 1 depicts demographics and intraoperative findings for each patient. The study population consisted of 22 patients: 12 patients who underwent tonsillectomy using 1940-nm diode laser and 10 patients using monopolar electro-cautery. Age and gender were not different between two groups. The mean operation time was similar as 21.8 minutes in laser group and 23.5 minutes in monopolar group. Moreover, the surgeon's subjective scales for intraoperative bleeding and cutting capacity were not significantly different between two groups. In laser group, bipolar electro-cauterization was used in 4 out of 12 cases compared to 9 out of 10 cases in monopolar group, which did not show a significant difference.

Pain scores of laser group were $4.67 \pm 1.97,2.92 \pm 1.51$, and $0.75 \pm 0.75$ at postoperative one day, one week, and two weeks, respectively. Pain scores of electro-cautery group were $4.60 \pm 0.97,4.90 \pm 2.18$, and $1.70 \pm 1.06$ at postoperative one day, one week, and two weeks, respectively. Especially, the laser group showed significantly lower score than electrocautery group on postoperative one week ( $p=0.001$ ) (Fig. 2A).

In the surgeon's assessment of scar contraction and swelling on postoperative one day, one week, and two weeks, scar contraction scores of laser group and electro-cautery group were $3.67 \pm 1.30,2.83 \pm 0.94$, and 1.33 $\pm 0.78 ; 3.60 \pm 1.17,3.00 \pm 0.82$, and $1.50 \pm 1.08$ at postoperative one day, one week, and two weeks, respectively. Swelling scores of laser group and electro-cautery group were $4.00 \pm 2.00,1.92 \pm 1.56$, and $0.50 \pm 0.52 ; 3.60 \pm 1.90$, $2.40 \pm 1.43$, and $0.50 \pm 0.53$ at postoperative one day, one week, and two weeks, respectively. There were no significant differences based on scar contraction and swelling scores between the two groups ( $p=0.885, p=0.546$ ) (Fig. 2B and $\mathrm{C}$.

The histological evaluation of the resected tonsils showed that the tonsil of laser group exhibits much smoother and undisrupted cytoplasm in H\&E stain (Fig. 3) and less destruction of collagen in trichrome staining (Fig. 4), compared to that of electro-cautery group.

\section{DISCUSSION}

Among number of complications that arise from tonsillectomy, postoperative pain stands as the major problem that jeopardizes the patient's quality of life; many instruments and different techniques have been developed and applied. ${ }^{10}$ With aforementioned idea of lower temperatures diminishing postoperative pain, laser has been taking center stage in surgical fields, along with harmonic ultrasonic scalpel and the coblator. Both cutting and coagulating ability of laser occur through absorption of laser energy by tissue, which is then converted to heat. Chromophores in tissues, such as water and hemoglobin, govern the quantity of absorption of laser energy. ${ }^{9}$ Absorption
A

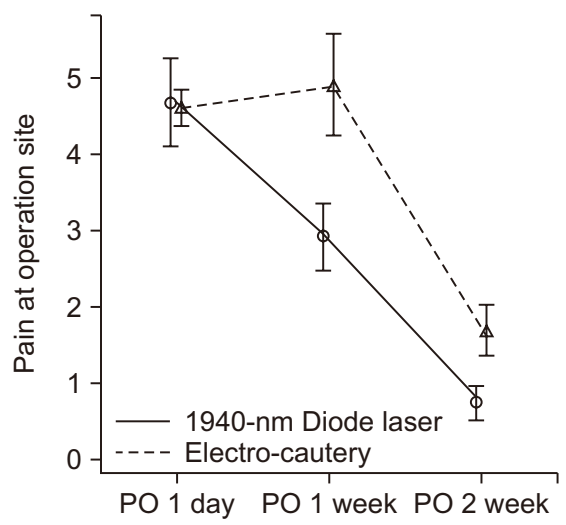

B

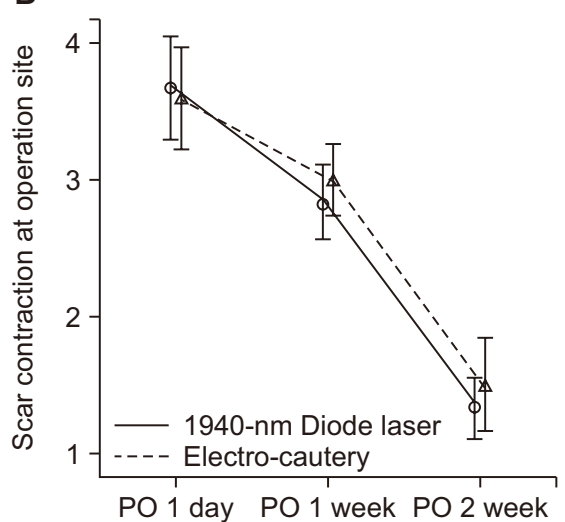

C

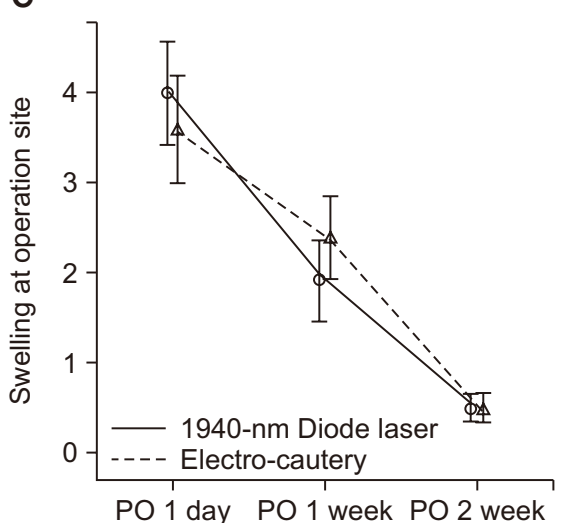

Fig. 2. Postoperative score on pain, scar contraction, and swelling. (A) Pain score on postoperative 1 day, 1 week, and 2 weeks. It shows significant decrease of pain score of 1940-nm diode laser group on postoperative 1 week compared to that of electro-cautery group. (B) Scar contraction on postoperative 1 day, 1 week, and 2 weeks. It shows no significant difference between 1940-nm diode laser group and electro-cautery group. (C) Swelling score on postoperative 1 day, 1 week, and 2 weeks. No significant difference between patients of 1940 nm diode laser group and bovie electro-cauterization group was noted. 

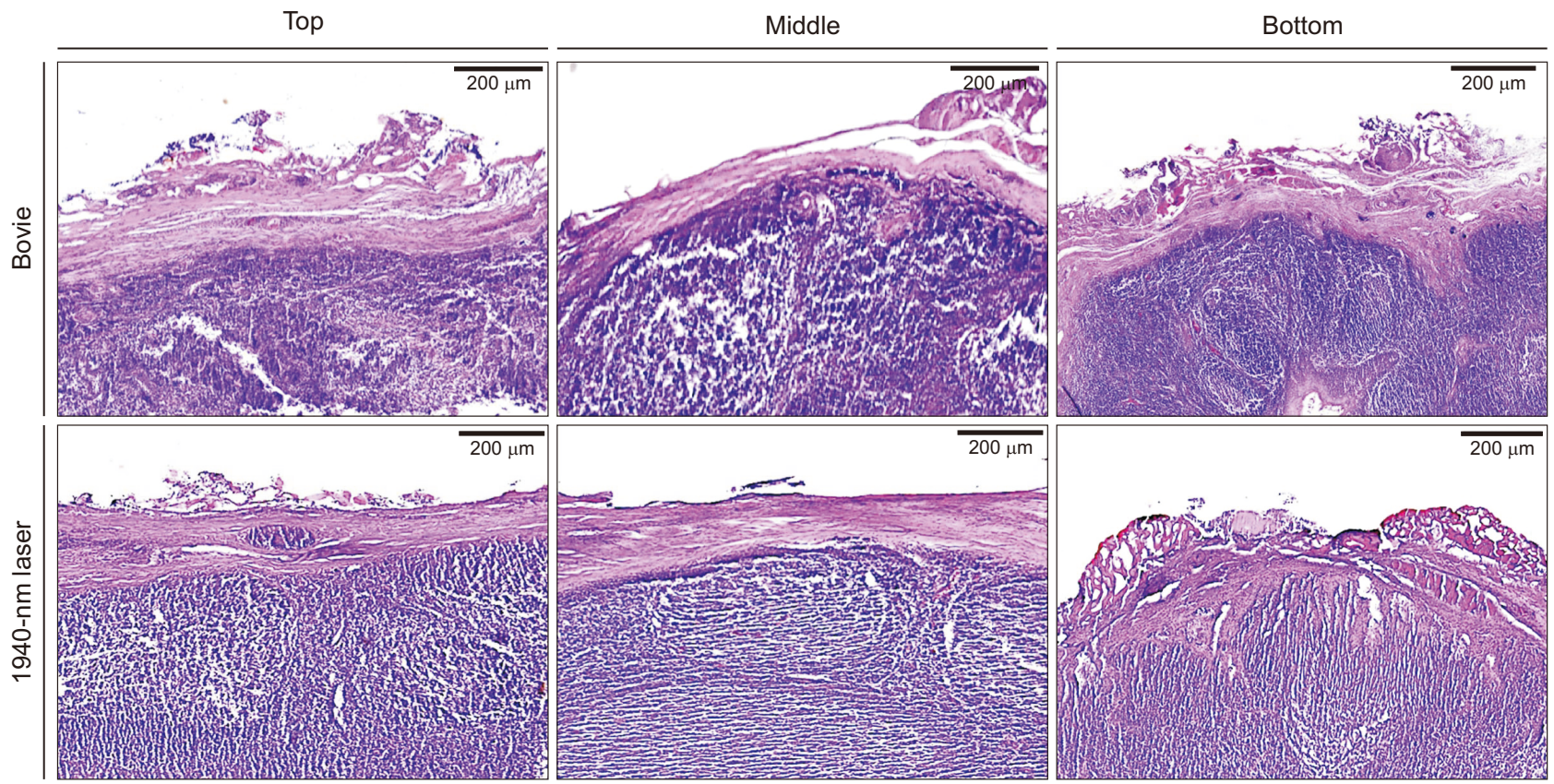

Fig. 3. Histological section of tonsils resected by electro-cauterization (Bovie) and 1940-nm diode laser (H\&E stain). (A, D) Refers to top section of the tonsil. (B, E) Refers to middle section of the tonsil. (C, F) Refers to bottom section of the tonsil. Histology of tonsil resected by 1940-nm diode laser displays much smoother and undisrupted cytoplasm.

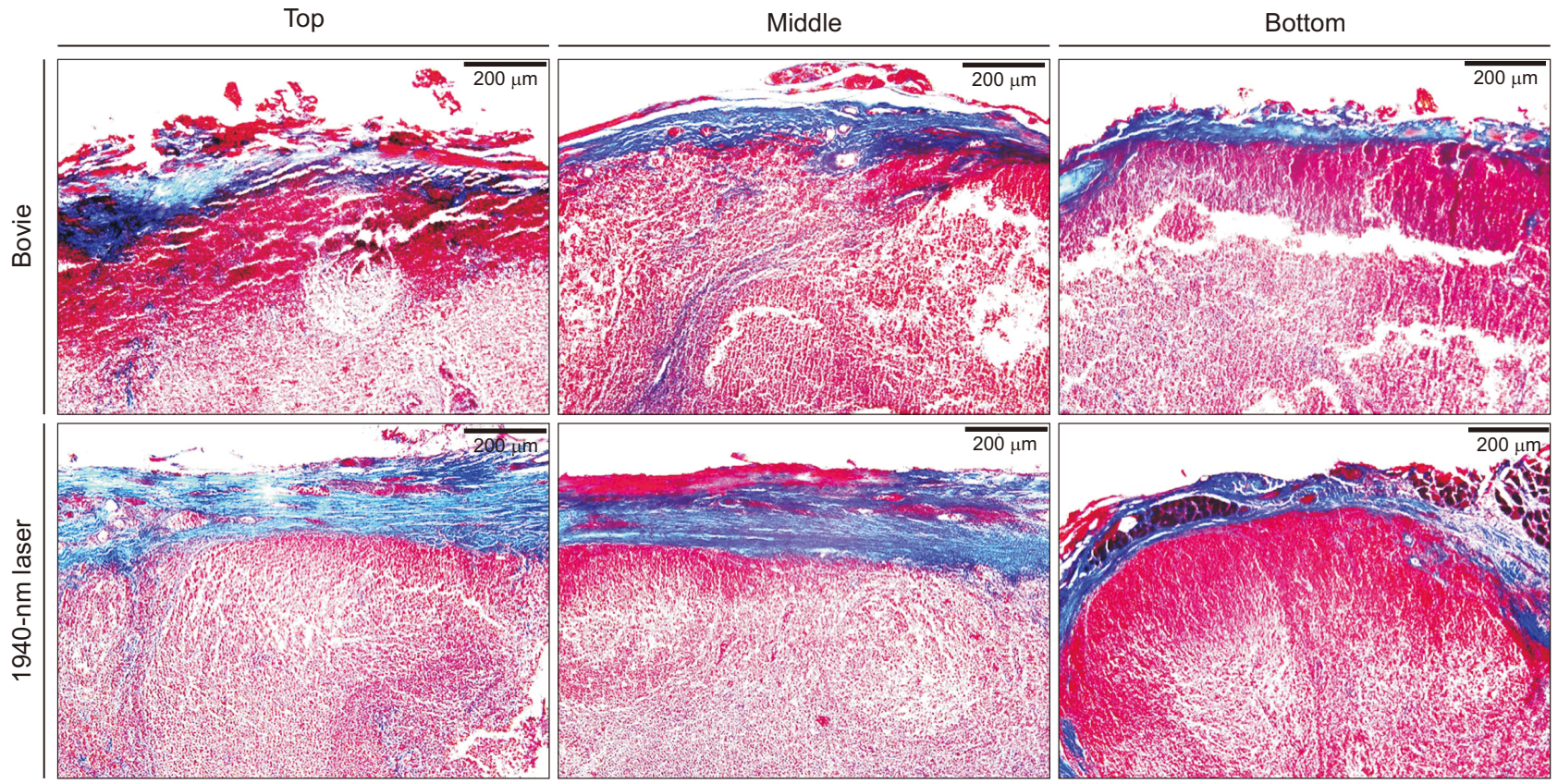

Fig. 4. Histological section of tonsils resected by electro-cauterization (Bovie) and 1940-nm diode laser (Trichrome stain). (A, D) Refers to top section of the tonsil. (B, E) Refers to middle section of the tonsil. (C, F) Refers to bottom section of the tonsil. Histology of tonsil resected by 1940$\mathrm{nm}$ diode laser displays less destruction of collagen.

peaks of hemoglobin lie in visible region of the spectrum, whereas absorption peaks of water lie in the infrared region. Therefore, our study focused on laser wavelength with both high water and hemoglobin absorption to provide sufficient cutting and coagulating power, respectively. The 1940-nm diode laser used in this study was origi- 
nally designed for body and facial lipolysis. With higher fat and water absorption than the conventional lasers of 980 , 1064,1320 , and 1440-nm, the 1940-nm diode laser was thought to have sufficient ability in tissue ablation with the benefit of excellent hemostasis that are well suited for tonsillectomy. Indeed, this study demonstrated excellent intra-operative performance and postoperative outcomes of 1940-nm diode laser. The operation time through this device was short, and satisfactory cutting capacity was proven well. In particular, prominent reduction of pain in postoperative one week in the group using 1940-nm diode laser compared to group using electro-cautery device was demonstrated.

According to several studies regarding laser and histology, wounds formed by laser behave differently compared to those formed by the scalpel; adjacent tissue receive minimal damage. ${ }^{11}$ In addition, the acute inflammatory reaction is minimal, along with few myofibroblasts in the wound during healing process, leading to little contraction and minimal scarring, thus resulting in lesser pain. ${ }^{12}$ Histological sections of tonsils resected by 1940-nm diode laser demonstrated much smoother and undisrupted cytoplasm, as well as less destruction of collagen. This result can be related to the wavelength of $1940-\mathrm{nm}$, which allows shallow and precise penetration depth, leading to accurate ablation with low thermal damage to adjacent tissues. ${ }^{13}$ These findings may have played an important role in reducing pain in postoperative one week.

As mentioned earlier, efficacies of laser in various field of medicine are already well proven. In particular, utilization of diode laser in urology and ophthalmology further suggest its ability to be exact and precise..$^{14}$ Moreover, additional reports of adequate tissue reduction with short operation time, diminished intra-operative and postoperative bleeding after using diode laser strengthen our result. $^{15}$

The major limitation of our study is small number of patients. However, this study is one of few that have considered evaluating the effectiveness of 1940-nm diode laser in tonsillectomy. Our results can be solid base for future research with larger cohorts to build upon.

\section{ACKNOWLEDGMENTS}

This research was supported by the Korea Health Technology R\&D Project (grant number: HI14C0748) through the Korea Health Industry Development Institute (KHIDI) by the Ministry of Health \& Welfare, and a Grant-Aid for Korea University Research and Business Foundation.

\section{REFERENCES}

1. Parsons SP, Cordes SR, Comer B. Comparison of posttonsillectomy pain using the ultrasonic scalpel, coblator, and electrocautery. Otolaryngol Head Neck Surg 2006;134:10613.

2. Timms MS, Temple RH. Coblation tonsillectomy: a double blind randomized controlled study. J Laryngol Otol 2002;116:450-2.

3. Sood S, Corbridge R, Powles J, Bates G, Newbegin CJ. Effectiveness of the ultrasonic harmonic scalpel for tonsillectomy. Ear Nose Throat J 2001;80:514-6, 518.

4. Khoder WY, Sroka R, Siegert S, Stief CG, Becker AJ. Outcome of laser-assisted laparoscopic partial nephrectomy without ischaemia for peripheral renal tumours. World J Urol 2012;30: 633-8.

5. Seitz M, Sroka R, Gratzke C, Schlenker B, Steinbrecher V, Khoder W, et al. The diode laser: a novel side-firing approach for laser vaporisation of the human prostate--immediate efficacy and 1-year follow-up. Eur Urol 2007;52:1717-22.

6. Khoder WY, Zilinberg K, Waidelich R, Stief CG, Becker AJ, Pangratz $T$, et al. Ex vivo comparison of the tissue effects of six laser wavelengths for potential use in laser supported partial nephrectomy. J Biomed Opt 2012;17:068005.

7. Mm J, Nk B, A P. Minimal intervention dentistry - a new frontier in clinical dentistry. J Clin Diagn Res 2014;8:ZE04-8.

8. Carruth JA. Resection of the tongue with the carbon dioxide laser. J Laryngol Otol 1982;96:529-43.

9. Guney M, Tunc B, Gulsoy M. Investigating the ablation efficiency of a 1940-nm thulium fibre laser for intraoral surgery. Int J Oral Maxillofac Surg 2014;43:1015-21.

10. Akural El, Koivunen PT, Teppo H, Alahuhta SM, Löppönen HJ. Post-tonsillectomy pain: a prospective, randomised and double-blinded study to compare an ultrasonically activated scalpel technique with the blunt dissection technique. Anaesthesia 2001;56:1045-50.

11. Frame JW. Removal of oral soft tissue pathology with the $\mathrm{CO} 2$ laser. J Oral Maxillofac Surg 1985;43:850-5.

12. Gabbiani G, Ryan GB, Majne G. Presence of modified fibroblasts in granulation tissue and their possible role in wound contraction. Experientia 1971;27:549-50.

13. Goharkhay K, Moritz A, Wilder-Smith P, Schoop U, Kluger W, Jakolitsch $\mathrm{S}$, et al. Effects on oral soft tissue produced by a diode laser in vitro. Lasers Surg Med 1999:25:401-6.

14. Silkiss RZ. THC:YAG nasolacrimal duct recanalization. Ophthalmic Surg 1993;24:772-4.

15. Janda P, Sroka R, Baumgartner R, Grevers G, Leunig A. Laser treatment of hyperplastic inferior nasal turbinates: a review. Lasers Surg Med 2001;28:404-13. 